\title{
HEALTHCARE WORKER READINESS TO ADOPT AND USE E-HEALTH TECHNOLOGY APPLICATIONS IN WESTERN KENYA
}

\section{ONDULO Jasper $^{1}$, ADUDA Dickens ${ }^{2}$, RAMBIM Dorothy ${ }^{1}$, OENGA Laban $^{1}$, KIMANZI Samuel ${ }^{1}$}

\author{
Department of Computer Science, School of Computing and informatics \\ Masinde Muliro University of Science and Technology \\ Kakamega, Kenya ${ }^{1}$ \\ School of Health Sciences \& Center for Research, Innovation and Technology, \\ Jaramogi Oginga Odinga University of Science and Technology, \\ Bondo, Kenya ${ }^{2}$ \\ Corresponding author: Jasper Ondulo. \\ Masinde Muliro University of Science and Technology
}

\begin{abstract}
Healthcare sector in general, more so in the developing world, is characterized by a number of constraints. Notably, available resources are always several paces behind the demands on healthcare. Adoption of e-health, however, enables increased access to health information and management of service delivery. While this is significant towards improving coordination and quality of health services, on the other hand, it increases the complexity of healthcare managements, resource needs as well as challenges of health information security. In Kenya, one of the recently introduced policy objective is to plan, design and install Information and Communications Technology (ICT) infrastructure, and software for the management and delivery of essential healthcare [1]. There is still dearth of information on the e-health readiness of health service providers to engage in actualizing this objective. The aim of this study was to investigate the underlying factors that affect healthcare workers readiness to adopt and use e-health technology applications in Kenya. Three County-referral health facilities located in 3 Counties in Western Kenya were identified based on availability, organization and functional scope of the e-health solutions. Using a cross-sectional descriptive design, quantitative data was collected from a total of 100 health service providers, through close-ended questionnaires and qualitative data from 21 key informants using interview guides and observation check lists. Available eHealth infrastructure and technical support were largely through donor funding. The study investigated staffs ease and comfort of use of the systems available, need for a management support in the organization providing strong leadership and coordination; e-health system as an additional workload burden, also studied was if the staff had requisite skills needed for full-scale use and need to learn. The investigation, revealed that there is need for management support providing a learning environment, hence the staff developing the requisite skills for full-scale use. These findings indicate that while basic e-health infrastructure exists, most workers still lacked the skills necessary for full-scale use and exploitation of eHealth advantages. There was a general unmet need for training and capacity building in eHealth among healthcare workers, particularly among the clinical cadres (frontline health workers). Implementation of eHealth should integrate ongoing competency and skills improvement activities mainly for clinical teams.
\end{abstract}

\subsection{BACKGROUND}

While eHealth plays a key role in enhancing the quality of health care management decisions at all levels of the health system [2]; its implementation in the developing countries is characterized by widespread multi-level disparities; which are thought to impact disproportionately on the health systems improvement interventions as well as their uptake [3; 4]. It is important to note that a number of factors interact in different ways to influence the adoption and implementation of e-health. The authors of "Towards a Digital Health Curriculum for Health Workforce for the African Region"; are of the view that, shortages of health informatics experts is one of the challenges hindering the training and recruitment of the health workforce. This, they opine is attributable largely to inadequate emphasis in the eHealth strategies of training of health workforce in health information management and computer technology. These are required to implement, operate and maintain a national eHealth system [4]. Requisite skills and expertise for data management, health languages and terminologies are the basis of electronic health records and health statistics. Without an adequate supply of qualified Health Information Managers and Health Information professionals, Government eHealth initiatives will fail and other health information applications will come under increasing threat [5]. Another issue which is evident from literature, is 


\section{International Journal of Advanced Research in Computer and Communication Engineering}

Vol. 10, Issue 3, March 2021

DOI 10.17148/IJARCCE.2021.10309

the poor usability of eHealth solutions, mirrored in data demand and information use, including analytics which are often a result of the users levels of training and expertise. The widespread multi-level disparities thus to a large extent, affects eHealth implementation programs in the developing countries. It is thus important to note at this point that due to this kind of a challenge, most of the implementation activities are at their lowest uptake and growth, due to health workforce phobia in handling computer systems related issues. Therefore, there is need to address these disparities in order to harness, integrate and grow eHealth operations into the health systems. This will require inter-sectoral collaboration, commitment and strategic planning focused on; besides building the physical infrastructure; deploying appropriate eHealth services and applications, emphasizing on developing a capable health workforce, achievable through appropriate training. Health professionals are the first to be concerned by changes brought about through eHealth solutions. However, often they are ill-prepared for such change, particularly as training programmes are often rudimentary or totally lacking. Further, they may also dread significant change out of a fear of losing authority or recognition. However it is important to note that an active collaboration of health professionals is an essential component in the implementation and maturity of eHealth solutions. The impediments to sharing information create unnecessary risks of error, inefficiency, and potential disparities in patients' outcomes. Limitations in the timely electronic movement of clinical information may mean that the key information about a patient whose risks have been identified, may not be captured appropriately and in a timely manner; more so, transferring the same information to other settings. It was noted that difficulties in obtaining information can mean that up-to-date evidence and best practice approaches do not always influence either clinicians' decisions or patients' choices about how to manage their health.

Additionally, eHealth fundamentally comprises five components: Telecommunication, Computing, medical equipment, Infrastructures and Infostructure [6]. This paper, however focused on Infostructure; which addresses, the human resources, organizational and administrative structures, policies, regulations, and incentives. Infostructure facilitates fully integrated and sustainable use of innovative ICTs and services to improve health care in an organized response to health and health care needs, issues, and challenges [7]. Progress in these fundamental areas will enable Kenya move closer towards having a health system that is sustainable, affordable, publicly funded and delivers excellent quality health services to its citizens.

\subsection{METHODOLOGY}

Health systems are complex, spanning clinical, administrative and financial transactions, such as billing and ordering; besides health-related information available to consumers and health professionals via the Internet. The study thus purposed to unveil the role of healthcare workforce, their competencies and skills in accessing, analyzing and using (data demand and information use), the myriad sources of information; besides sharing with different cadre of people who may need it and when they need it in electronic form. To achieve the objectives of the study, the researchers, first identified three study sites which differed in locale and a number of structural settings, namely, the sites exhibited urban, semiurban and rural setting. This was to help bring out clearly whether the type of setting had any effect on the workforce engagement in eHealth implementations and its maturity. Therefore, the researchers purposed to do a mixed methodology study approach. A numerical observational study to gather relevant timestamps, administration of questionnaires and a number of qualitative interviews to construct a better view of the situation.

\subsection{Data Collection of Quantitative Data}

The study applied hand delivery method. Trained research assistants hand delivered the questionnaires with the help of contact persons at each study site. The respondents, who participated in the study from the three study sites, were a broad mixture of medical professionals, systems and human resource administrators. This composition gave a good crosssection of care providers (healthcare workforce) which was the aim of the study. Care was taken to ensure that each of the various groups contained a range of participants from different backgrounds and a mix of diverse range of age cadre. The broad range of respondents was deemed appropriate to contribute to the generalizability of the results.

To understand the workflow processes and problems faced on the ground, a state analysis was performed. A global assessment of all work processes and a time motion study was conducted for one week at each of the selected study sites. This allowed for the analysis and understanding of the bottlenecks and problem areas within the patient flow, the role of the workforce and their interaction with technology in this process. This entailed characterization of patient flow to determine the points of start and end, integration of information technology; and other patterns of movements within the flow. Conventional events within the patient flow process were taken into consideration (Registration, billing, triage, provider/patient consultation, diagnosis (Laboratory, Pharmacy and Radiology), staff scheduling and loading, etc. When this had been done, it was mapped against the service charter to compare and contrast for the variations whether positive or negative, more so in the context of use of or non-use of and integration of eHealth systems in the daily work-flow. Additionally, department data was requested as background information and to supplement the observational data and interviews. 


\subsection{Data Collection of Qualitative Data}

The researchers, conceptualized what they perceived to be the interacting variables, informed by a theoretical framework which they had developed and validated. These were then used to define the topics and questions covered during qualitative data collection. Within each study site, purposive sampling strategy was adopted to obtain maximum variability, key informants were identified; with a focus to include implementers from three levels (top level management, middle level management and operational level). Health staff with responsibility in the organizations, middle management, including IT leads, training leads and others with day-to-day responsibility for the implementation issue under study; at least a doctor, clinician and a nurse practitioners were interviewed; besides IT experts and administrators. An interview guide for providers was structured to contain questions about the influence of external (environmental) factors, organizational characteristics, processes (both social and technological) and clinical routines on service delivery. The qualitative method was used to explain and validate quantitative results from the questionnaire phase. Interview questions were formulated after an analysis of the response of the quantitative phase. Personal interviews were conducted in the form of semi-structured interviews. This allowed researchers to encourage conversations with participants, and give participants the flexibility to elaborate on information which they thought were relevant to the research topic. Further the researchers also had the freedom to navigate during the interviews, moving away from scripted questions, allowing the participants to lead the interview in the direction of their own interests and personal constructs. Definite questions were given to potential participants in advance so that they would be aware of the types of questions that would be asked. The interviews were held in the work area of interest. Data was collected through face-to-face interviews with staffs identified through the purposive sampling framework. The interviews were used to determine not only what happened, but also the implementers' perceptions on why it happened. There were about 21 respondents from the three study sites who were interviewed on various issues pertaining to e-health implementation, health workers' e-readiness, and their role in its successful implementation, integration and embedding the daily routine healthcare processes. The main issues emerging from the qualitative study were thematically analyzed.

\subsection{RESULTS}

A total of 100 respondents returned duly filled questionnaires, besides 21 respondents who were interviewed from the three study sites, giving a total of 121 respondents for both quantitative and qualitative data: from study site A (40); from study site B (41); from study site C (40). At least $40 \%$ had been in employment before computers were introduced in the respective health facilities.

Staff distribution: nurses were the majority at 38\%, followed by general administrative staff at 20\%, clinicians at $15 \%$, medical doctors at $6 \%$, pharmacists at $6 \%$, laboratory technicians at $7 \%$, radiologists at $6 \%$ and network/system administrators at $2 \%$

Perceived level of comfort with the use of computer-based technology: at least $22 \%$ of the respondents of the sample were anxious, $26 \%$ responded as moderate, $33 \%$ were generally at ease, while $19 \%$ were completely at ease. Males were more at ease in using computer-based technology than were the female staffs. In addition, $12 \%$ of nurses were anxious in the use of computer-based technology, followed by the clinicians at $8 \%$; the doctors were either at ease at $2 \%$ or completely at ease at $4 \%$. Of the administrators' perceived comfort with the use of computer-based technology, $6 \%$ reported moderate comfort, $12 \%$ at ease, while $2 \%$ were completely at ease. All the systems administrators were completely at ease with computer-based technology (mainly because this is their core training). Of the pharmacists, $2 \%$ were anxious, $2 \%$ moderate, $1 \%$ at ease and $1 \%$ completely at ease. Of Laboratory technicians $3 \%$ were moderately at ease and $4 \%$ at ease with use of computer-based technology. Radiologists on the other hand were $3 \%$ moderate, $2 \%$ at ease and $1 \%$ completely at ease with the computer-based technology.

Organizational factors that facilitates service providers' participation or taking active role in the use of eHealth: the respondents responded to the question on: $i$ ) the 'need for Management Support (providing strong Leadership and coordination)'; $i$ i) the need for Management Support (providing learning environment); iii) perception of e-health system as an additional workload burden. At least $38 \%$ agreed and $62 \%$ strongly agreed that there is need for a management support in the organization providing strong leadership and coordination. On the need for Management Support (providing learning environment) $6 \%$ were undecided, 53\% agreed, while $41 \%$ strongly agreed that there is need for management support providing a learning environment. Regarding perception of e-health system as an additional workload burden, $29 \%$ did not agree, $54 \%$ were undecided, while $17 \%$ felt that the system creates additional workload.

Compatibility of current staff skills and workload with existing E-health systems: of the respondents, $65 \%$ were undecided, while $35 \%$ agreed that there was compatibility. As for learnability of new skills required to use the system, the study found out that $54 \%$ were undecided, $35 \%$ agreed on the need for new skills to use the system; while $11 \%$ 


\section{International Journal of Advanced Research in Computer and Communication Engineering}

Vol. 10, Issue 3, March 2021

DOI 10.17148/IJARCCE.2021.10309

strongly agreed. The respondents were also asked on their perception on the impact of the system on the distribution of responsibilities between individuals. Only 3\% disagreed on the efficiency resulting due to usage of the system, $42 \%$ remained undecided, and 53\% agreed that using the system results into efficiency and 2\% strongly agreed on Individuals' perception of the efficiency of using the system.

Coherence and collective action were assessed given their potential to be disruptive to the systems processes. From the results, $53 \%$ of the respondents were undecided while $47 \%$ agreed that there was impact on the distribution of responsibilities between individuals by using the system.

How eHealth systems has impacted on individuals' perception of liability: $33 \%$ were undecided, $65 \%$ agreed and $2 \%$ strongly agreed that using the system impacted on individuals' perception of liability. Further, the study also sought to know whether eHealth systems had impact on quality. Slightly more than half of the respondents seem to agree on the perceived impact of the system on the quality of the interaction between professionals and patients: That is to say $49 \%$ agreed plus $2 \%$ who strongly agreed. The remaining $49 \%$ were undecided.

Ease of using the system is often believed to be a determinant in the adoption and implementation of an innovation and eventually its integration and embedding in the routine work; thus, the research sought to establish how the staff perceived the system. The responses were $3 \%$ of the respondents disagreed, while $36 \%$ were undecided, $49 \%$ agreed that it was easy using the system and $12 \%$ strongly agreed.

Collective action and coherence: this was based on respondents' response about their perceived existence of a shared understanding of what e-health system is for and how it is to be used: $57 \%$ were undecided, while $43 \%$ agreed. The response shows lack of e-health system at the facilities. Additionally, collective action is a fabric that holds the system together; thus, it was important to understand the commitment of individuals to making the system work which can be seen as a strand of the bigger set. To this question, $5 \%$ of the respondents disagreed and 58\% were undecided on the commitment of individuals to make the system work while $37 \%$ agreed.

Qualitative results: Key findings were that staff training, cost of training and quality of training programs in addition to staff apathy to change due to unfamiliarity with computers or fear of computers, and lack of training during basic training/exposure at the college were cited as major impediments to implementation process since few staffs are trained on e-health modules and services.

\subsection{DISCUSSIONS}

The current study findings indicate important human-resource challenges in the readiness of healthcare workers to adopt and use eHealth at facility levels in Kenya. Less than half of the service providers were able to use the computer-based technologies with ease. Majority of them had a 'felt' need for availability of management support with regard to: $i$ ) strong leadership and coordination of the activities; ii) providing a learning environment to enhance their computer and information skills; iii) mitigating perceived additional workload-related burden associated with e-health systems activities. This may indicate a general unmet need for training and capacity building in eHealth among healthcare workers, particularly among the clinical cadres (frontline health workers), who not only are the majority of healthcare workers in Kenya but also would play a critical role in transforming access, care delivery, patient experiences, and health outcomes through electronic health (eHealth).

In a patient-centered healthcare setup, the frontline healthcare workforce forms a formidable strand in supporting critical service delivery activities and enabling them flow smoothly [1]. This however remains true only when the workforce are supported with the right skills and expertise. From the data gathered during the study, about $40 \%$ of current workers were in the workforce before the proliferation of technology, thus lacked requisite training, and hence most had anxiety or phobia for the information technology and the related innovations. It can therefore be argued that, lack of training and/or preparation with computer skills hinder the adoption of technology and lead to cognitive and attitudinal barriers. The high proportion of 'undecided', phobic or anxious groups may indicate ambivalence or lack of engagement by healthcare workers whose core responsibilities are not routinely computer-based. However, there is need to further investigate the latent issues underlying these findings.

Furthermore, previous studies in different contexts have shown that integration of information system into workflow may create additional workload burden until users are routinized and the systems embedded into the routine workflow. The responses of perceived increased workload attributable to eHealth activities obtained in this study are consistent with ehealth systems which have not yet matured to be routinized and be embedded into the daily workflow. In Kenya, eHealth notably has not matured compared to the developed economies hence a sizeable percentage of healthcare workforce 


\title{
International Journal of Advanced Research in Computer and Communication Engineering
}

\author{
Vol. 10, Issue 3, March 2021
}

DOI 10.17148/IJARCCE.2021.10309

remains phobic or anxious in using or applying them routinely in their daily work, while in addition, there is concurrent lack of leadership [8].

\subsection{CONCLUSIONS}

There exist in this study a critical unmet need in adoption of eHealth among frontline service providers whose core responsibilities are clinical-based, due to a lack of basic computer and information technology skills. The availability of digital health infrastructure in the referral level health facilities in Kenya represents an opportunity for developing stronger health systems to ensure adequate service delivery by frontline health workers. Hence, there is need to simultaneously introduce improvement measures as adoption of computer-based technologies expands. This may include professional training to enhance cognitive capabilities (knowledge, attitude and skills) to enable them navigate the operations of the eHealth systems at all levels within their work-related routines. Thematic challenges emerging, related to both systems factors and individual provider factors, indicates potential complex causes of lack of service provider readiness that call for multilevel interventions.

One key challenge to leveraging IT in health or its effective implementation, is the need for a skilled workforce that understands healthcare, information and communication technologies, and the human and organizational challenges involved. Decision makers must therefore consider: identifying, developing and embodying, the skills, training, and competencies - consistent with local cultures, languages, and health systems - that will be needed to realize the full benefits of these technologies. Further, they must be prepared to take a leadership role in disseminating best practices by facilitating communication and supporting discussion among healthcare providers regarding meaningful use.

\section{REFERENCES}

[1]. MoH. 2016. Kenya National eHealth Policy 2016-2030.

[2]. Jones G, Steketee RW, Black RE, Bhutta ZA, (2003): Morris SS; Bellagio Child Survival Study Group. How many child deaths can we prevent this year? Lancet.; 362 (9377):65-71.

[3]. Getnet Bogale Fanta et al., (2018): Economic analysis of sustainable eHealth implementation in developing countries: a systematic review.

[4]. Lackey, Suf an, Dzombak \& Mehta, "Demonstrating demand for preventive health services in rural Kenya" -Journal of Humanitarian Engineering, Vol 3 No 1

[5]. Michael Legg \& Associates, (2009); A review of the Australian health informatics workforce, report prepared for the Department of Health and Ageing, Melbourne, Health Informatics Society of australia in association. http://www.hisa.org.au/system/files/u2233/Australian_Health_Informatics_Workforce_Review_v1_1.pdf

[6]. Viziteu, L. (2008). Horizons and Perspectives eHealth. Analele Universității Din Craiova-Seria Științe , 1581-1591. Retrieved from http://www.ceeol.com/aspx/getdocument.aspx?logid=5\&id=eee42fcb194542599295caa8431876a0

[7]. Asamoah-Odei, E., Kebede, D., Zielinski, C., Soumbey-Alley, E.-W., Peixoto, M., \& Moeti, M. (2011). Leveraging eHealth to Improve National Health Systems in the African Region. Retrieved from http://www.aho.afro.who.int/sites/default/files/ahm/reports/47/ahm-issue-14-leveragingehealth.pdf

[8]. Ondulo Jasper, Aduda Dickens, Rambim Dorothy. (2020). Availability and Integration of e-Health Technologies in Routine Service Delivery in Western Kenya. E-ISSN 2278-1021/ ISSN 2319-5940 International Journal of Advanced Research in Computer and Communication Engineering, Vol. 9, Issue 6, June 2020. DOI 10.17148/IJARCCE.2020.9619 112 